\title{
Aging-associated oxidized albumin promotes cellular senescence and endothelial damage
}

\author{
This article was published in the following Dove Press journal: \\ Clinical Interventions in Aging \\ 29 February 2016 \\ Number of times this article has been viewed
}

\author{
Carlos Luna ${ }^{1, *}$ \\ Matilde Alique ${ }^{2, *}$ \\ Estefanía Navalmoral ${ }^{2}$ \\ Maria-Victoria Noci ${ }^{3}$ \\ Lourdes Bohorquez-Magro² \\ Julia Carracedo' \\ Rafael Ramírez ${ }^{2}$ \\ 'Nephrology Unit, Instituto \\ Maimónides de Investigación \\ Biomédica de Córdoba (IMIBIC), \\ Reina Sofía University Hospital, \\ Córdoba, Spain; ${ }^{2}$ Department of \\ Systems Biology, Physiology Unit, \\ Universidad de Alcalá, Madrid, \\ Spain; ${ }^{3}$ Anesthesia Unit, Reina sofía \\ University Hospital, Córdoba, Spain \\ *These authors contributed equally \\ to this work
}

Correspondence: Matilde Alique Departamento Biología de Sistemas, Facultad de Medicina y Ciencias de la Salud, Universidad de Alcalá, Alcalá de Henares E-2887I, Madrid, Spain

Tel +34 9l 8856436

Email matilde.alique@uah.es

\begin{abstract}
Increased levels of oxidized proteins with aging have been considered a cardiovascular risk factor. However, it is unclear whether oxidized albumin, which is the most abundant serum protein, induces endothelial damage. The results of this study indicated that with aging processes, the levels of oxidized proteins as well as endothelial microparticles release increased, a novel marker of endothelial damage. Among these, oxidized albumin seems to play a principal role. Through in vitro studies, endothelial cells cultured with oxidized albumin exhibited an increment of endothelial damage markers such as adhesion molecules and apoptosis levels. In addition, albumin oxidation increased the amount of endothelial microparticles that were released. Moreover, endothelial cells with increased oxidative stress undergo senescence. In addition, endothelial cells cultured with oxidized albumin shown a reduction in endothelial cell migration measured by wound healing. As a result, we provide the first evidence that oxidized albumin induces endothelial injury which then contributes to the increase of cardiovascular disease in the elderly subjects.
\end{abstract}

Keywords: elderly, oxidative stress, microparticles, vascular damage

\section{Introduction}

Aging is associated with well-known changes in protein conformation that are involved in aging-related disease. Among this modification, probably the protein oxidation is the most relevant mechanism of pathogenesis in the elderly subjects. Oxidative modifications generally cause loss of catalytic or structural function in the affected proteins; it is likely that the level of oxidatively modified proteins observed during aging will have serious deleterious effects on cellular and organ function. ${ }^{1,2}$ Although the oxidative damage to nucleic acids is subject to repair by highly efficient excision/insertion mechanisms, the repair of damaged to proteins appears limited to the reduction of oxidized derivatives. ${ }^{3,4}$ Damaged proteins are targeted for degradation to amino acid constituents by the action of various endogenous proteases, especially the 20 s proteasome. ${ }^{3,4}$ However, the age-related accumulation of oxidized proteins may reflect age-related increases in rates of reactive oxygen species (ROS) generation, decreases in antioxidant activities, or losses in the capacity to degrade oxidized proteins. ${ }^{2}$ Therefore, the importance of protein oxidation in aging is supported by the observation that levels of oxidized proteins increase with subject age. ${ }^{5}$

ROS can react directly with the protein or they can react with others molecules (such a sugars and lipids). This generates products which then react with the protein. Many of reactions are mediated by free radicals. ${ }^{6}$ Proteins are major targets for ROS because of their abundance in biological systems. In addition, proteins are primarily responsible for most functional processes within the cells. The major protein present in the plasma is albumin, which constitutes $\sim 55 \%$ of the plasma proteins. ${ }^{7}$ As a result, it is most susceptible BY
hereby accept the Terms. Non-commercial uses of the work are permitted without any further permission from Dove Medical Press Limited, provided the work is properly attributed. For permission for commercial use of this work, please see paragraphs 4.2 and 5 of our Terms (https://www.dovepress.com/terms.php). 
to suffer an oxidative process. ${ }^{8}$ In this manner, the oxidation of albumin may cause endothelial damage. Nevertheless, there are no studies analyzing the effects of oxidized albumin in aging, and as a consequence endothelial damage.

It is now recognized that the oxidative modification of proteins by reactive species, especially ROS, is implicated in the etiology or progression of an important number of diseases. ${ }^{9}$ Compared to control samples, proteins are more oxidized in tissues of animals and patients suffering from Alzheimer's disease, rheumatoid arthritis, atherosclerosis, or amyotrophic lateral sclerosis. ${ }^{10}$ Oxidized proteins are also associated with aging-related diseases and diabetes, ${ }^{5,11}$ neurodegenerative diseases (Alzheimer's), ${ }^{9,12}$ and cardiovascular diseases among others. ${ }^{13-15}$ In addition, cardiovascular diseases show a significantly elevated mortality in elderly patients and have been associated with endothelial cell injury. Furthermore, cardiovascular diseases have been proven to cause a decline in endothelial function. ${ }^{16}$ Specifically, age-related endothelial dysfunction has been characterized in animals and humans. ${ }^{17}$ In addition, oxidized proteins have been demonstrated to be a critical contributor to the development of atherosclerosis, contributing to the formation, progression, and complications of atherosclerotic plaques. ${ }^{18}$ Noteworthy, in another study, oxidized proteins lead to endothelial dysfunction. ${ }^{19}$ As a result, there is great interest in studying new target therapies to prevent or reverse the aging-induced oxidative stress in endothelial cells.

Furthermore, microparticles (MPs) have been used as biomarkers of cells damage and activation. ${ }^{20,21}$ MPs are a heterogeneous population of small membrane fragments shed from various cell types. The endothelium is one of the primary targets of circulating MPs, and MPs isolated from blood have been considered biomarkers of vascular injury and inflammation..$^{202-24}$ Endothelial damage and the release of membrane MPs are key steps in the pathogenesis and development of some diseases associated with damaged vasculature. ${ }^{25}$ In this regard, adhesion molecules, including intercellular adhesion molecule-1 (ICAM-1) and vascular cell adhesion molecule-1 (VCAM-1), are secreted by activated endothelial cells in atherosclerotic lesions, stimulating immune cell and monocyte recruitment and migration into the intimal area of the vascular wall. ${ }^{26,27}$ Besides, endothelial cell apoptosis is also implicated in a number of cardiovascular conditions. ${ }^{27}$

Several studies support a role of cellular senescence in aging-associated diseases. ${ }^{28}$ Cellular senescence is a process in which cells cease dividing and undergo distinctive phenotypic alterations. Senescent endothelial cells are usually observed in a wide variety of diseases such as cardiovascular diseases. Senescence contributes to the overall decline in tissue regenerative potential that occurs with aging.
Furthermore, an accelerated senescence in endothelial cells has been proposed to explain the premature apparition of cardiovascular diseases.

As a result, this study has determined whether the levels of oxidized proteins in sera may be involved with markers of endothelial damage associated with aging. In addition, the study also determines whether the aforementioned damage may be induced by the oxidation of the albumin.

\section{Materials and methods Patients and sample collection}

Study population

Participants in the study were recruited from the Alcala University. All participants were volunteers who had submitted written informed consent to participate in the study and advance approval was obtained from the ethical committee of the Universidad de Alcalá. The study was performed in accordance with the World Medical Association Declaration of Helsinki. In all cases, the subjects have clinical history of age-related diseases such as type 2 diabetes mellitus, cardiovascular disease, renal disease, or cancer.

The study population included 16 subjects. The control group consisted of seven young healthy subjects aged between 28 and 31 years and who were comparable in sex and with nonsmoking habits. The elderly group consisted of eight healthy subjects aged between 75 and 82 years and who were also comparable in sex and with nonsmoking habits.

\section{Plasma extraction}

Peripheral blood samples were collected from patients in the morning using EDTA-coated tubes. Samples were centrifuged for 15 minutes at 1,200 rpm. Plasma was transferred into a new tube and stored at $-20^{\circ} \mathrm{C}$ until use.

\section{Culture cells}

Primary human umbilical vein endothelial cells (HUVECs; ATTC Cat Number PCS-100-010) were cultured in endothelial growth media (EGM) from Lonza (Basel, Switzerland), supplemented with $10 \%$ FBS (heat inactivated). Culturing conditions were $37^{\circ} \mathrm{C}, 5 \% \mathrm{CO}_{2}$, and $95 \%$ humidity. HUVECs were used for experiments between passages 2 and 7. Previously, HUVECs were serum-deprived overnight before treatments.

\section{Determination of endothelial microparticles (EMPs) in plasma and endothelial cells}

\section{Plasma}

Platelet-free plasma was obtained by centrifugation at $1,500 \mathrm{rpm}$ for 10 minutes at room temperature, followed by an additional centrifugation at 15,000 rpm for 30 minutes in 
order to separate the MPs. MPs were resuspended in PBS to determinate the number of endothelial microparticles (EMPs) as described in the following section.

The MPs from plasma were incubated with monoclonal antibody against phycoerythrin (PE)-labeled anti-CD31 (as an endothelial marker; BD Bioscience, San Jose, CA, USA), followed by incubation with fluorescein isothiocyanateconjugated (FITC) Annexin-V kits according to the manufacturer's instructions (BD Bioscience). The negative control was obtained using the anti-isotype antibodies. An equal volume of flow count calibrator beads (Beckman Coulter, Marseilles, France) were added. Fluorescence-activated cell sorter analysis was performed in an Accuri C6 flow cytometer (BD Bioscience).

\section{Endothelial cells (HUVEC)}

Culture supernatants from endothelial cells treated with native and oxidized albumin for 24 hours were collected and cleared from detached cells and cell fragments by centrifugation at 1,500 rpm for 10 minutes at room temperature. The supernatants were then subjected to centrifugation at 15,000 rpm for 30 minutes. Pelleted EMPs were resuspended in PBS and then quantified. An equal volume of flow count calibrator beads (Beckman Coulter) was added. The EMPs in HUVEC were determined and quantified in an Accuri C6 flow cytometer (BD Bioscience) using forward scatter intensity value (size) and side scatter intensity value (granularity).

\section{Preparation of oxidized albumin}

Albumin (Albutein 20\%; GRIFOLS, Barcelona, Spain) was diluted to $0.1 \mathrm{mg}$ protein $/ \mathrm{mL}$ with EDTA-free PBS and incubated with $\mathrm{CuSO}_{4}(10 \mu \mathrm{mol} / \mathrm{L})$ for 18 hours at $37^{\circ} \mathrm{C}$. At the end of incubation, $0.1 \mathrm{mmol} / \mathrm{L}$ EDTA was added to prevent further oxidation. ${ }^{29}$ Oxidized albumin was diluted to $20 \mathrm{mg} / \mathrm{mL}$ in PBS. Either native albumin or oxidized albumin was added at a final concentration of $2 \mathrm{mg} / \mathrm{mL} .^{30}$

\section{Determination of plasma AOPPs}

Plasma concentrations of advanced oxidation protein products (AOPPs) were measured in duplicate using a commercially available ELISA kit (CUSABIO, Wuhan, Hubei, People's Republic of China, Cat Number CSB-E09925h) following the manufacturer's protocol.

\section{Protein carbonyl assay (OxyBlot)}

OxyBlot analysis was performed, according to manufacturer's specifications (Millipore, Billerica, MA, USA), to identify carbonyl groups that are introduced into the amino acid side chain after oxidative modification of proteins. The level of protein oxidation was determined by an Oxidized Protein Detection Kit (OxyBlot, Chemicon, Billerica, MA, USA, Cat\# S7150-Kit). The OxyBlot kit derivatizes carbonyl groups to a 2,4-dinitrophenylhydrazone (DNP) moiety. The DNP moiety can then be detected using anti-DNP antibodies and is a method to assay for one form of oxidative damage to a protein. The proteins were derivatized as per the protocol given in the kit. These proteins were separated on $10 \%$ SDS-PAGE gels and transferred membranes were washed in washing buffer (PBS with $0.2 \%$ Tween-20). Protein bands were visualized with Luminata Crescendo Western HRP Substrate (Millipore).

\section{Sample preparation and bidimensional electrophoresis (2D PAGE)}

The samples used (native and oxidized albumin) for the identification of carbonylated proteins were prepared for analysis by two-dimensional gel electrophoresis.

First, both native and oxidized albumin were precipitated with acetone overnight at $-20^{\circ} \mathrm{C}$. The following day, aliquots of solubilized samples containing $500 \mu \mathrm{g}$ of proteins were mixed with sample buffer containing $30 \mathrm{mM}$ DTT, $0.5 \%$ v/v ampholytes (Biolytes pH 3-10; Bio-Rad, Hercules, CA, USA), and $0.001 \% \mathrm{w} / \mathrm{v}$ bromophenol blue to a total volume of $300 \mu \mathrm{L}$ and loaded on IPG strips (17 cm, pH range: 4-7; Bio-Rad) for protein electrofocusing in a Protean IEF cell system (Bio-Rad). Strips were passively rehydrated for 2 hours at $20^{\circ} \mathrm{C}$, and then the voltage was gradually increased $(30-8,000 \mathrm{~V})$ till it reached $60,000 \mathrm{Vh}$.

Prior to the separation of proteins according to their molecular weight, strips were equilibrated for 20 minutes in equilibration buffer (6 M urea, $1.5 \mathrm{M}$ Tris- $\mathrm{HCl} \mathrm{pH} 8.8$, $2 \%$ SDS, and 20\% glycerol) containing 2\% DTT, followed by 20 minutes in equilibration buffer containing $135 \mathrm{mM}$ iodoacetamide. Second-dimension separation was performed in polyacrylamide Mini-Protean TGX precast gel (BioRad) using a Mini-Protean tetra cell (Bio-Rad). Gels were run at a constant current intensity of $60 \mathrm{~mA}$ during 3 hours. A total of two gels were performed for each condition from two different biological samples.

\section{Gel staining, image acquisition, and analysis}

Two-dimensional gels were stained with Coomassie brilliant blue G-250 (Bio-Rad) for 24 hours (according to the study by Herbert et al), ${ }^{31}$ and then destained by washing with $0.1 \mathrm{M}$ Tris $-\mathrm{H}_{3} \mathrm{PO}_{4}(\mathrm{pH} 6.5$ ) for 5 minutes, followed by 1 minute with $25 \% \mathrm{v} / \mathrm{v}$ methanol and finally 5 minutes with $20 \% \mathrm{w} / \mathrm{v}$ ammonium sulfate. Gels were digitized using a 
GS-800 calibrated densitometer (Bio-Rad) and the images analyzed with the PDQUEST software 8.0.1 (Bio-Rad), using spot-by-spot manual matching between the studied conditions.

\section{Endothelial adhesion molecules expression}

Expression of VCAM-1 and ICAM-1 were measured from endothelial cells treated with vehicle and different doses of native and oxidized albumin for 24 hours. After treatment, HUVECs were obtained by mechanical disruption and washed once with PBS $1 \times$. Next, $10 \mu \mathrm{L}$ VCAM-1 (CD106-PE conjugate, BD Pharmingen [BD Pharmingen, San Diego, CA, USA]) and $10 \mu \mathrm{L}$ ICAM-1 (MHCD5401FITC conjugate, Invitrogen, Waltham, MA, USA) antibodies were used to assess the expression in the different experimental conditions. HUVECs were incubated with the antibodies for 20 minutes in darkness. Then, cells were washed with PBS $1 \times$ and fixed with BD CellFIX ${ }^{\mathrm{TM}}$ (Becton Dickinson, Cat Number 340181 [Becton Dickinson Bioscience, San José, CA, USA]). Finally, we proceeded to the data acquisition in the cytometer, with HUVECs without antibody labeling used as a reference (as a negative control). We performed the experiment in duplicates $(n=3)$. For the analysis of data acquired in the cytometer, we used the mean fluorescence intensity (MFI) of different antibodies (VCAM-1-PE and ICAM-1-FITC).

\section{Apoptosis quantification}

The percentage of apoptosis was measured by annexin- $\mathrm{V}$ and propidium iodide (PI) staining of endothelial cells treated with vehicle and different doses of native and oxidized albumin for 24 hours. HUVECs were obtained by mechanical disruption and washed once with annexin-V binding buffer (FITC Annexin-V Apoptosis Detection Kit I, Becton Dickinson). Cells were then suspended in $195 \mu \mathrm{L}$ of annexin-V binding buffer and $5 \mu \mathrm{L}$ of annexin-V (556419, Becton Dickinson) and PI (51-66211E, Becton Dickinson) were added following the manufacture's protocol. The negative tube controls that did not contain either annexin-V or PI were placed with $195 \mu \mathrm{L}$ of annexin-V binding buffer. Cells were incubated for 15 minutes in darkness. Next, the cells were washed once with buffer and finally suspended in $490 \mu \mathrm{L}$ of buffer. The samples were acquired within 1 hour in flow cytometer (FACSCalibur, Becton Dickinson). We performed this experiment in duplicates $(n=3)$. For the analysis of the data acquired, cell populations that were positive for both annexin-V and PI were utilized. These cells were considered to be in late apoptosis state. Finally, we analyzed the differences, in percentages, obtained in the experimental conditions.

\section{Detection of ROS}

Hydroethidine (Invitrogen), a substance that is oxidized by ROS to become ethidium, which emits red color, was used to measure superoxide anion. The HUVEC monolayer was incubated in EGM medium, with vehicle, native albumin, and oxidized albumin at different doses $(\mathrm{mg} / \mathrm{mL})$, and without FBS for 4 hours at $37^{\circ} \mathrm{C}$ with $\mathrm{CO}_{2}$. At the end of the treatments, the cells were exposed for 15 minutes at $37^{\circ} \mathrm{C}$ to $2 \mu \mathrm{M}$ hydroethidine (HE). Analyses were performed in a flow cytometer (FACSCalibur, Becton Dickinson). Intracellular ROS production was measured as a percentage of positive cells marked with HE.

\section{Senescence assay}

HUVECs were cultured in their own medium with the different treatments. Cells were fixed at $70 \%$ confluence and then incubated at $37^{\circ} \mathrm{C}$ overnight with the staining solution containing the X-gal substrate (Catalog \#JM-K320-250; Senescence Detection kit, MBL International Corp, Woburn, MA, USA). Cells were then observed under a microscope for development of blue color.

\section{Wound healing assay}

HUVECs were seeded in six-well plates $\left(1 \times 10^{5}\right.$ cells/well), incubated in medium with 10\% FBS for 24 hours, and subsequently washed twice with PBS and incubated in medium with $0 \%$ FBS. After the cells grew to confluence, a straight line was scratched across the culture with a $10-200 \mu \mathrm{L}$ micropipettor tip. The cells were treated with the different treatments and were incubated at $37^{\circ} \mathrm{C}$ and $5 \% \mathrm{CO}_{2}$. Images of the cells were obtained with an inverted phase contrast microscope at different times. The wound width was determined with ImageJ software (National Institutes of Health, New York, NY, USA).

\section{Statistical analysis}

Data are presented as mean \pm SD. Significance of differences from control values was determined with the Student's $t$-test, with two-tailed distribution between groups as indicated in Table 1 (AOPPs ELISA). Mean separations were performed with one-way analysis of variance, with Bonferroni correction used for the in vitro experiments. A value of $P<0.05$ was considered to indicate statistical significance. All statistical tests were performed with GraphPad Prism software version 5.0 (Graph Pad Software, Inc., La Jolla, CA, USA). 
Table I AOPPs and levels of endothelial microparticles in the plasma of healthy young subjects compared with healthy elderly subjects

\begin{tabular}{|c|c|c|c|c|c|c|}
\hline Subjects & Age & Sex & AOPP $(\mathrm{ng} / \mathrm{mL})$ & Median \pm SD & EMPs $(\mu L)$ & Mean \pm SD \\
\hline I & 29 & Male & 11.84 & $8.33 \pm 4.70$ & 4 & $14.86 \pm 9.32$ \\
\hline 2 & 30 & Male & 13.47 & & 6 & \\
\hline 3 & 28 & Female & 13.17 & & 16 & \\
\hline 4 & 29 & Male & 9.68 & & 29 & \\
\hline 5 & 30 & Female & 9.27 & & 8 & \\
\hline 6 & 31 & Female & 9.82 & & 18 & \\
\hline 7 & 28 & Male & 5.23 & & 23 & \\
\hline 8 & 75 & Male & 28.95 & $30.64 \pm 12.74 *$ & 99 & $109.1 \pm 42.96 *$ \\
\hline 9 & 82 & Female & 21.53 & & 62 & \\
\hline 10 & 81 & Female & 47.33 & & 122 & \\
\hline II & 79 & Female & 51.95 & & 72 & \\
\hline 12 & 77 & Male & 39.27 & & 83 & \\
\hline 13 & 76 & Female & 22.16 & & 104 & \\
\hline 14 & 80 & Female & 14.30 & & 143 & \\
\hline 15 & 79 & Male & 23.61 & & 203 & \\
\hline 16 & 81 & Male & 26.59 & & 94 & \\
\hline
\end{tabular}

Notes: Concentration of AOPPs is expressed as $\mathrm{ng} / \mathrm{mL}$ of chloramine-T equivalents and EMPs amount is expressed as number of microparticles per $\mu \mathrm{L}(\mathrm{EMPs} / \mu \mathrm{L}) . * \mathrm{P}<0.00 \mathrm{I}$ versus young subjects. In the case of AOPPS, all the values are median \pm SD and mean \pm SD for amount of EMPs ( $n=7-9$ per group, respectively).

Abbreviations: AOPPs, advanced oxidation protein products; EMPs, endothelial microparticles; SD, standard deviation.

\section{Results}

\section{Increase of AOPPs plasma levels in aging subjects compared with young subjects (controls)}

Plasma protein oxidation and its correlation to human aging were analyzed in this study. Oxidized plasma proteins were evaluated as AOPPs by ELISA. Plasma concentration levels of AOPPs from aging subjects were significantly increased compared with young subjects, who comprised the control group (Table 1). Therefore, we observed that the levels of oxidized proteins increase with subject age.

\section{EMPs rise in elderly subjects}

There are studies that show the MPs ratio as a marker of the endothelial damage. ${ }^{20,21}$ To study the role of MPs ratio in the aging process, we measured the number of EMPs in the plasma of different subjects by flow cytometry. As a result, we observed that there was an incremental increase in the amount of EMPs in the elderly subjects compared with young subjects (Table 1). This finding implicates that the increase of EMPs is associated with aging.

\section{Determination of albumin oxidation in vitro}

To analyze the biological effect of oxidized albumin on the endothelial cells, we utilized the albumin oxidation. The albumin oxidation with $\mathrm{CuSO}_{4}$ procedure was confirmed by different techniques. First, the albumin oxidation was confirmed by OxyBlot analysis. The amount of oxidation was proportional to the signal intensity and we found a higher OxyBlot band in the oxidized albumin versus native albumin (Figure 1A). We also probed the albumin oxidation using 2D PAGE technique. The gel with oxidized albumin presented a clear difference in the position of albumin spots (Figure 1B). In addition, the 2D PAGE analysis revealed (as shown in Figure 1B) a change in the $\mathrm{pI}$ of oxidized albumin (6.8) of -1.5 units with respect to native albumin pI (8.3).

\section{Oxidized albumin increases adhesion markers in a dose-response effect in endothelial cells}

To quantify the endothelial damage in vitro, we measured some typical markers of adhesion, ${ }^{32,33}$ such as VCAM-1 and ICAM-1, in endothelial cells (HUVECs). We performed a dose-response treatment with native and oxidized albumin for 24 hours in serum-deprived HUVEC. We observed an increase in VCAM-1 expression (Figure 2A) when treating with different doses of oxidized albumin for 24 hours. Furthermore, we observed increased levels of ICAM-1 (Figure 2B) in a dose-response pattern after a 24-hour exposure period with oxidized albumin treatment versus treatment with native albumin and vehicle. These observations suggest that treatment with oxidized albumin activates the endothelial cells.

\section{Oxidized albumin enhances the apoptosis levels in endothelial cells}

It has been described that apoptosis plays an important role in the endothelial damage. ${ }^{34,35}$ To check the possibility that 


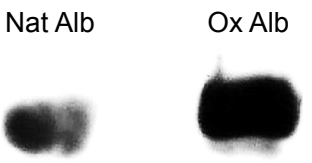

B

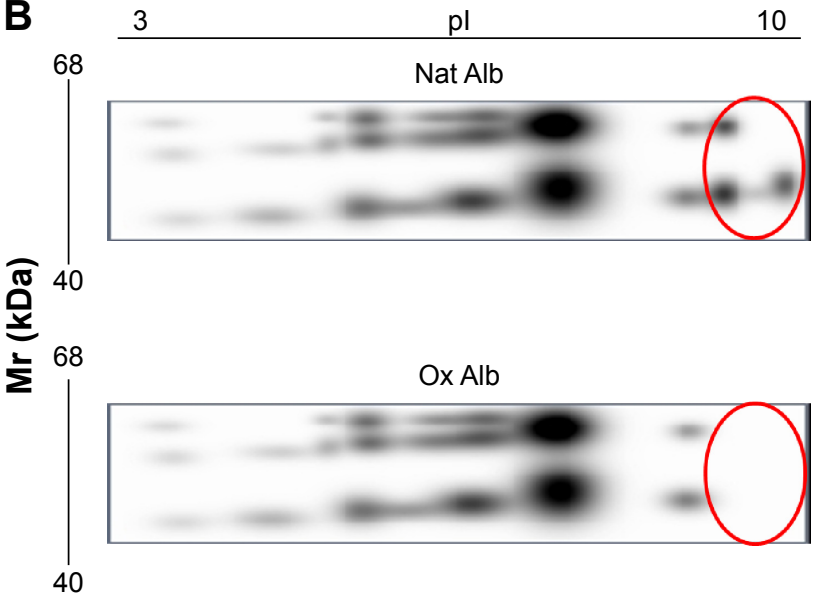

Figure I Confirmation of the albumin oxidation.

Notes: (A) OxyBlot technique for detection of carbonyl groups. The carbonyls generated by $\mathrm{CuSO}_{4}$ were detected by reaction with DNPH and anti-DNP immunostaining (OxyBlot). The analyses show one major band corresponding to Ox Alb. (B) Two-dimensional gels of native and oxidized albumin produced from TCA precipitation. IEF separation of proteins was achieved using $7 \mathrm{~cm}$ IPG strips, $\mathrm{pH} 3-10$, and the second dimension was run on a $10 \%$ polyacrylamide gel. (Upper) Proteome of the Nat Alb of the TCA precipitation and (lower) proteome of oxidized albumin. Images are representative of experiments performed on native and oxidized albumin samples $(\mathrm{n}=2)$.

Abbreviations: DNPH, 2,4-dinitrophenylhydrazine; DNP, 2,4-dinitrophenyl; TCA, Trichloroacetic acid; Mr, relative molecular mass; IPG, immobilized $\mathrm{pH}$ gradient; $\mathrm{Ox}$ Alb, oxidized albumin; Nat Alb, native albumin

oxidized albumin produces endothelial damage via apoptosis, we measured the apoptosis levels with different concentrations of native and oxidized albumin (Figure 3A) in resting HUVECs. After a 24-hour exposure period, we observed upregulated apoptosis levels in a dose-response effect with the oxidized albumin treatment compared with different native albumin concentrations and vehicle. This confirms that another marker of endothelial damage is modified with oxidized albumin treatment.

\section{Oxidized albumin treatment elevates the EMPs production in supernatants of endothelial cells}

As mentioned before, there exists a relationship between the number of EMPs in plasma and the aging process, which is exhibited by the elevated levels of oxidized protein. We also performed studies in vitro to measure the numbers of EMPs generated by oxidized albumin. The results showed an increase in the production of EMPs with different doses of oxidized albumin treatment, whereas there was no change in the EMPs production with different concentrations of native albumin compared with vehicle supernatants cells (Figure 3B). Thereupon, as in the case of plasma samples, there is also a link between the number of EMPs and the oxidized protein amount.

\section{Oxidized albumin generates oxidative stress}

To test the possibility of oxidized albumin increasing ROS production, we have used a $\mathrm{HE}$ as a probe for measurement of intracellular ROS. Resting HUVECs were treated with and without different doses of native and oxidized albumin for 4 hours and vehicle. Flow cytometry intracellular ROS production (Figure 4B) and the total ROS production (Figure 4C) were significantly higher after oxidized albumin treatments compared with the native albumin and vehicle cells. Surprisingly, we also observed that the native albumin treatments
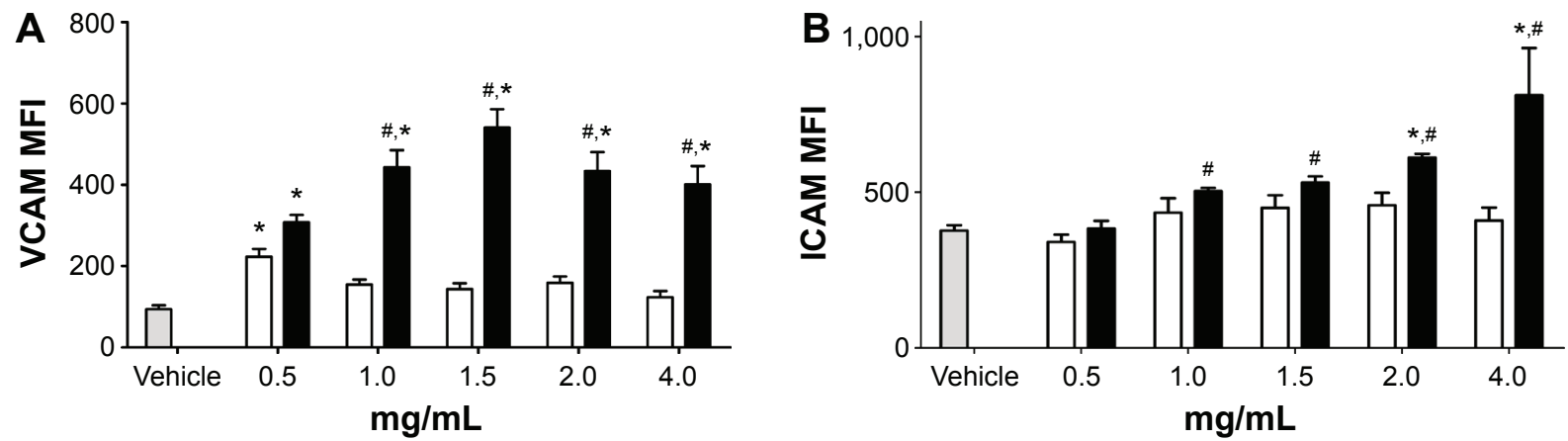

Nat Alb

Ox Alb

Figure 2 Flow cytometer analysis of endothelial adhesion molecules, (A) VCAM-I and (B) ICAM-I, expression in $\mathrm{HUVEC}$ incubated with vehicle CuSO ${ }_{4}(\mathrm{I} 0 \mu \mathrm{mol} / \mathrm{L})+0 . \mathrm{I}$ mmol/L EDTA (gray bar), Nat Alb (white bars), or Ox Alb (black bars).

Notes: HUVEC were treated with different doses $(\mathrm{mg} / \mathrm{mL})$ of native and oxidized albumin for 24 hours. MFI are presented as mean $\pm S D, n=3$, in duplicate. $* P<0.05$ versus vehicle; ${ }^{\# P}<0.05$ versus at all Nat Alb doses.

Abbreviations: Ox Alb, oxidized albumin; Nat Alb, native albumin; MFI, median fluorescence intensity; HUVECs, human umbilical vein endothelial cells; VCAM-I, vascular cell adhesion molecule-I; ICAM-I, intercellular adhesion molecule-I; EDTA, ethylenediaminetetraacetic acid; SD, standard deviation. 


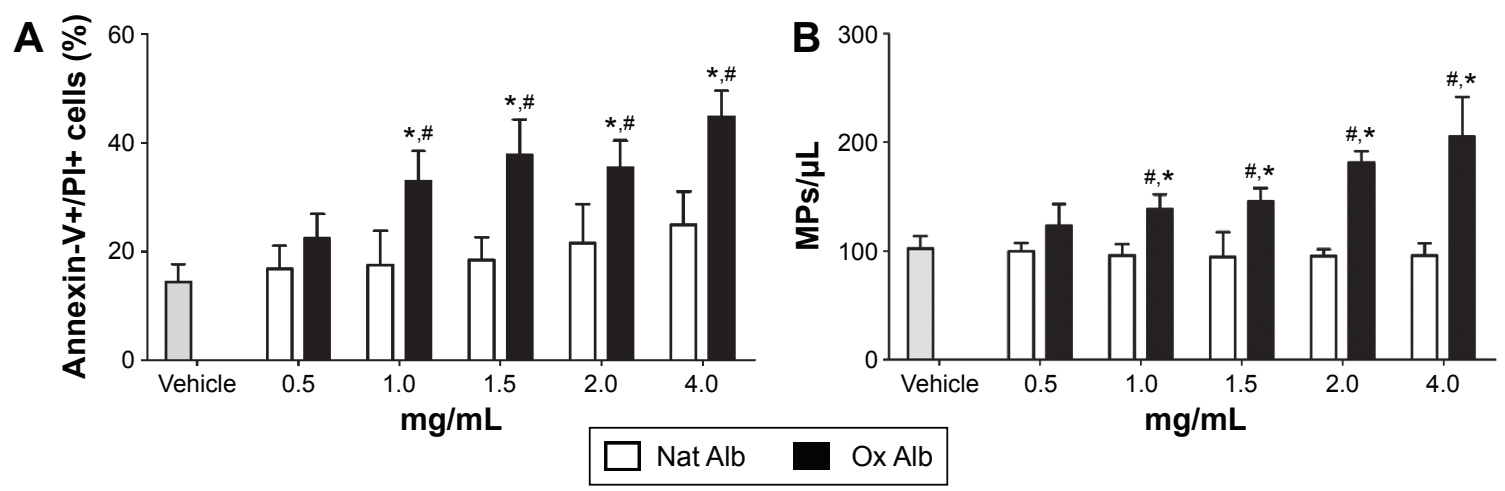

Figure 3 Flow cytometer determination of (A) cellular apoptosis and (B) endothelial microparticles expression in the supernatants of albumin-treated cells.

Notes: (A) Endothelial cells were treated with different doses of Nat Alb (white bars), Ox Alb (black bars), and vehicle CuSO ${ }_{4}(10 \mu \mathrm{mol} / \mathrm{L})+0.1 \mathrm{mmol} / \mathrm{LEDTA}$; (gray bar) for 24 hours. After incubation, apoptosis was determined in Pl-stained cells and analyzed by flow cytometry. Data are expressed as mean $\pm S D, n=3$ in duplicate. $* P<0.05$ versus vehicle; ${ }^{\# P}<0.00 \mathrm{I}$ versus at all Nat Alb doses. (B) Endothelial cells were treated with native (Nat Alb; white bars), oxidized albumin (Ox Alb; black bars), and vehicle $\mathrm{CuSO}_{4}(10 \mu \mathrm{mol} / \mathrm{L})+0.1 \mathrm{mmol} / \mathrm{LEDTA}$; (gray bar) for 24 hours at different concentrations. Total microparticles from HUVECs were characterized by flow cytometry. Sizeselected events were plotted as a function of their size and complexity, and also near of I $\mu \mathrm{m}$ flow count calibrator beads. Graphic shows the EMPs production per $\mu \mathrm{L}$ with the vehicle, native, and oxidized albumin treatment. Data are presented as mean \pm SD, $n=3$ in duplicate. ${ }^{*} P<0.05$ versus vehicle, ${ }^{*} P<0.05$ versus Nat $A$ Ilb.

Abbreviations: Ox Alb, oxidized albumin; Nat Alb, native albumin; HUVEC, human umbilical vein endothelial cells; EMPs, endothelial microparticles; Pl, propidium iodide; SD, standard deviation.
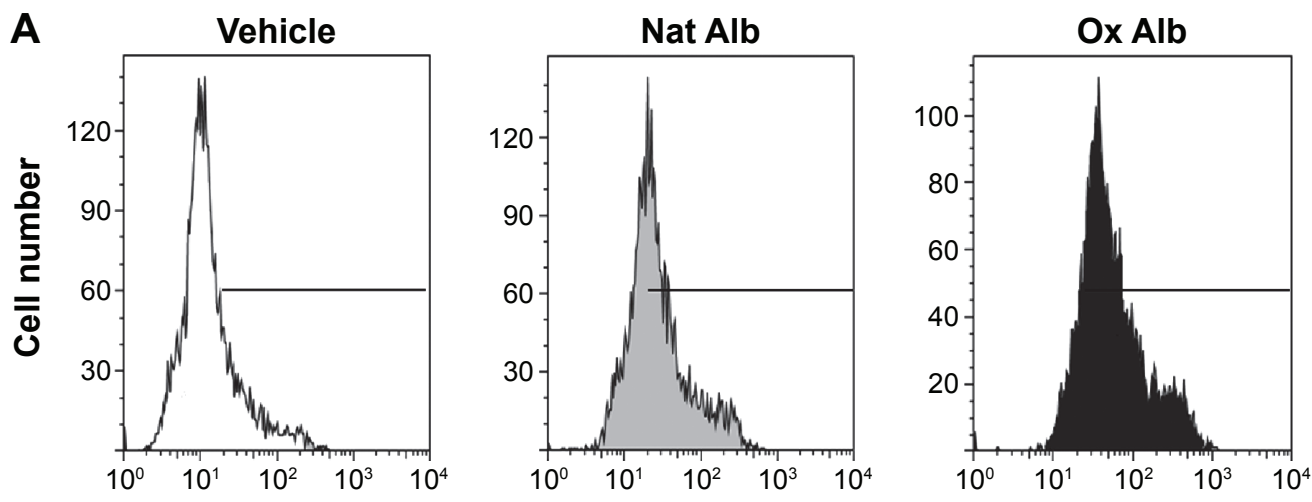

Reactive species oxigen (ROS) hydroethidine $(2 \mathrm{mg} / \mathrm{mL}$ )

B

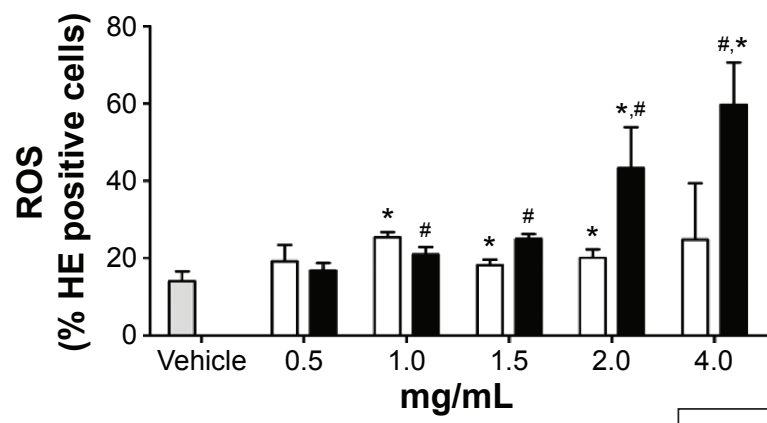

C

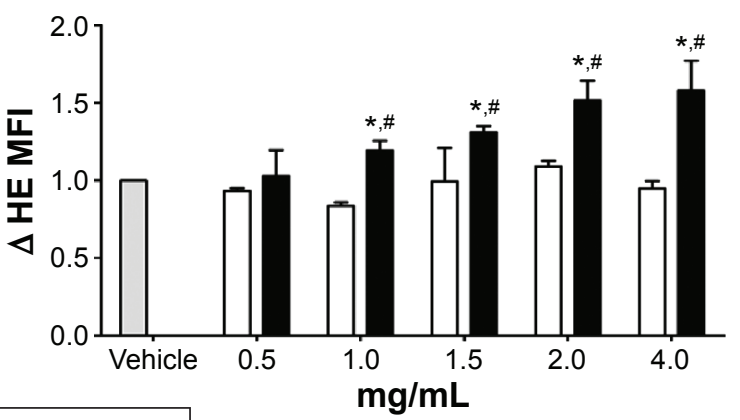

Nat Alb

Ox Alb

Figure 4 (A) Flow cytometer analysis of intracellular ROS production in HUVEC incubated with vehicle (white), Nat Alb (gray), or Ox Alb (black). HUVEC were treated with native and oxidized albumin $(2 \mathrm{mg} / \mathrm{mL})$ for 4 hours, (B) the histogram shows ROS as the percentage of HE-positive cells, and (C) MFI with vehicle (gray bar) and different doses of Nat Alb (white bars) and Ox Alb (black bars) for 4 hours.

Notes: Data are presented as mean $\pm S D, n=4$ in duplicate. $* P<0.05$ versus vehicle; ${ }^{*} P<0.05$ versus at all Nat Alb doses.

Abbreviations: Ox Alb, oxidized albumin; Nat Alb, native albumin; HUVEC, human umbilical vein endothelial cells; ROS, reactive oxygen species; HE, hidroethidine; MFI, median fluorescence intensity; SD, standard deviation. 
upregulated the percentage of ROS (Figure 4B) production versus vehicle-treated cells, whereas there were no differences in the total ROS production between native albumintreated cells and vehicle-treated cells (Figure 4C). Thereby, albumin oxidation is associated with oxidative stress due to endogenous production of ROS by mitochondria.

\section{Increment of senescent endothelial cells by albumin oxidation treatment}

To investigate the role of oxidized albumin in the senescence of endothelial cells, staining for senescence-associated $\beta$-galactosidase was carried out (Figure 5). Staining revealed that there was a 2.3 -fold increase in the number of $\beta$-galactosidase-positive cells in oxidized albumin-treated HUVECs (Figure 5B), whereas the value with native albumin was significantly lower and similar to vehicle cells. By morphological inspection, we found that senescent cells increase in size and display a more flat morphology than native albumin-treated HUVECs (Figure 5A). Importantly, the senescence effect observed in the oxidized albumintreated endothelial cells was reproducible with the replicative senescence (population doublings undergone by human endothelial cells) of HUVEC. ${ }^{36}$

\section{Oxidized albumin inhibits endothelial cell wound healing migration}

Finally, we studied migration associated with the albumin oxidation in endothelial cells. The wound healing migration assay illustrated a reduction in migration in oxidized albumin-treated HUVEC, whereas no significant changes were observed between native albumin-treated cells and vehicle-treated cells (Figure 6). Hence, the treatment with the oxidized albumin showed a reduction of migration activity in endothelial cells.
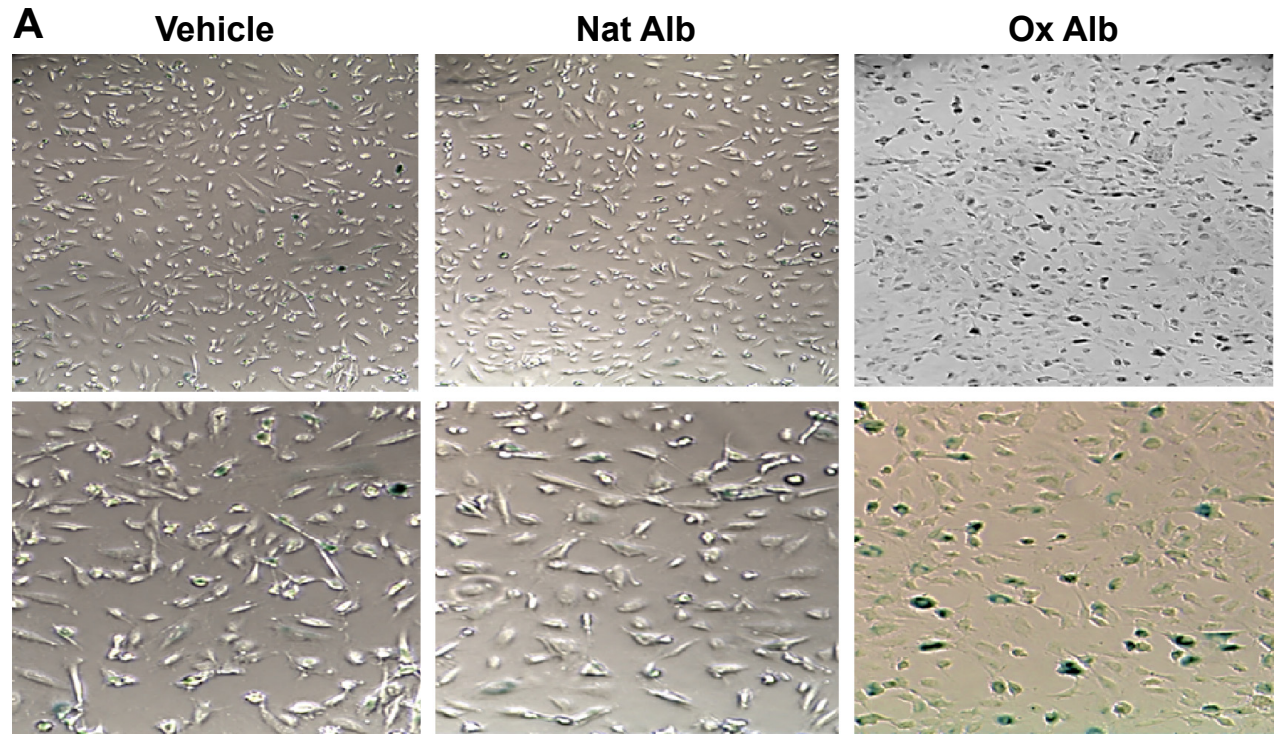

B

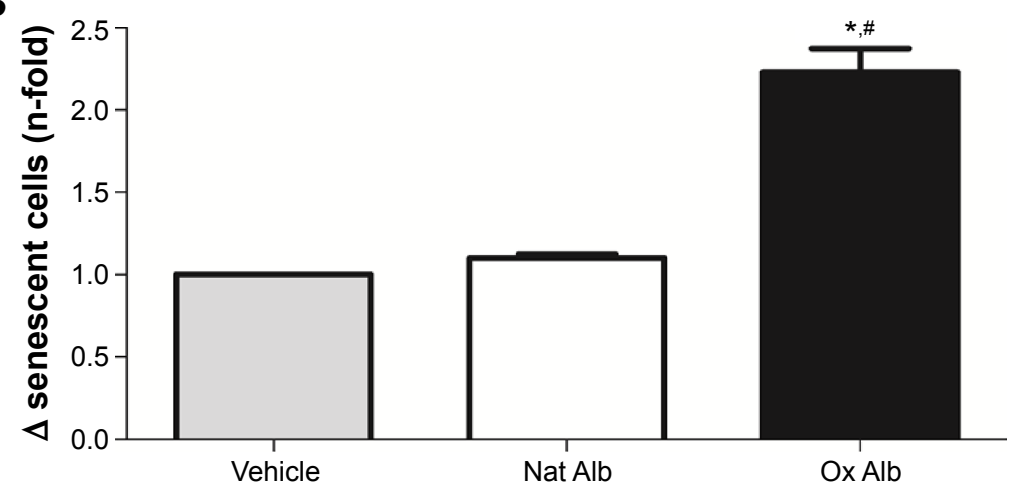

Figure 5 Senescent-associated $\beta$-galactosidase activity staining of native and oxidized albumin treatments.

Notes: HUVEC were treated with Nat Alb and Ox Alb $(2 \mathrm{mg} / \mathrm{mL})$ for 24 hours. (A) Representative pictures of cells stained with $\beta$-galactosidase (I0x). A zoom of each picture is showed. (B) Graphic shows $n$-fold increase in $\beta$-galactosidase-positive cells. Data are presented as mean $\pm S D, n=3$. $* P<0.05$ versus vehicle; ${ }^{*} P<0.05$ versus Nat Alb. Abbreviations: Ox Alb, oxidized albumin; Nat Alb, native albumin; HUVEC, human umbilical vein endothelial cells; SD, standard deviation. 

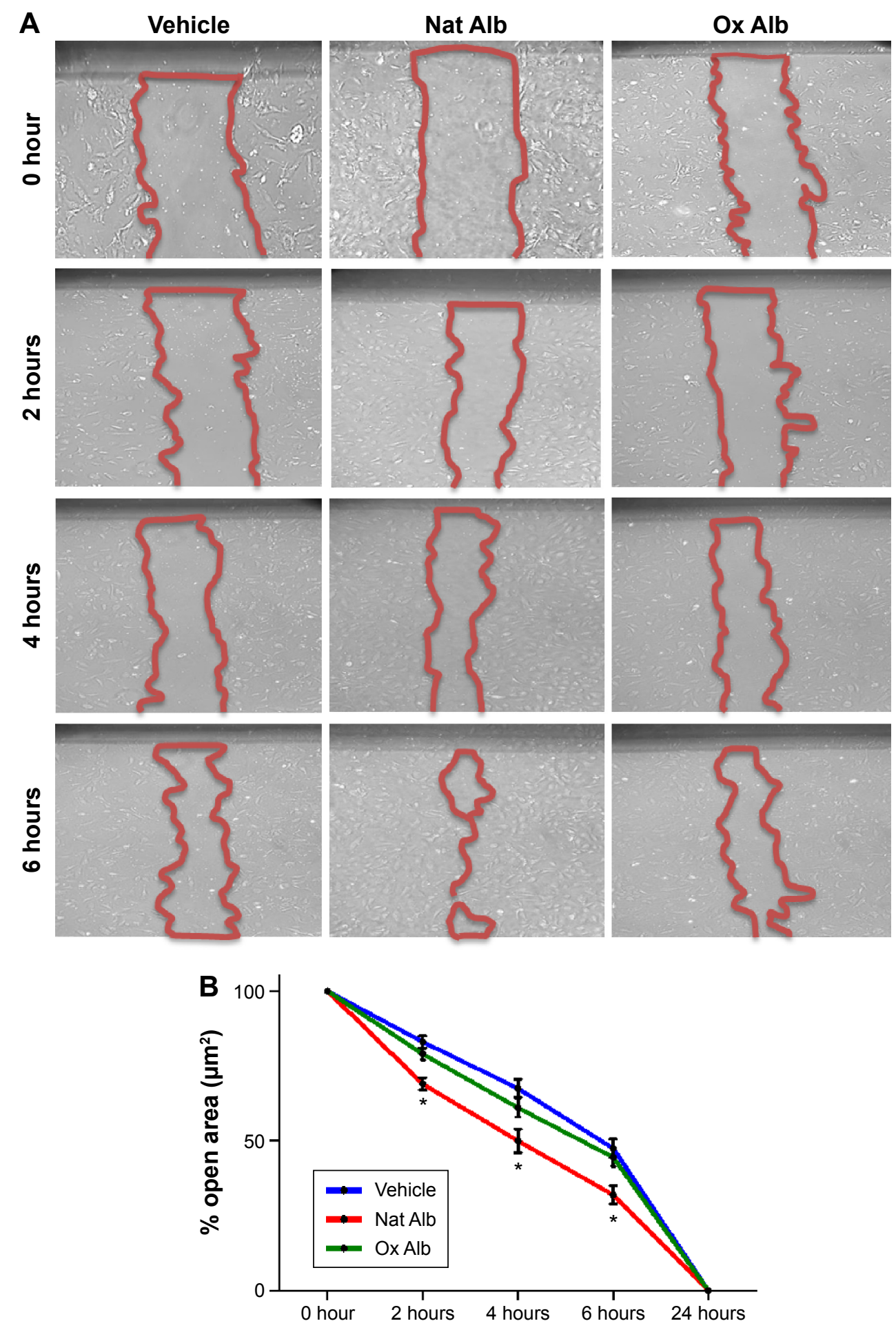

Figure 6 HUVEC with Nat Alb and Ox Alb $(2 \mathrm{mg} / \mathrm{mL})$ were scratched and wound margins were imaged from 0 up to 24 hours later.

Notes: Experiments were performed as in $(\mathbf{A})$, and the extent of wound closure was quantified by measuring the wound area and comparing with the initial wound area $(n=3)(B) . * P<0.05$ versus vehicle and $O x$ Alb at the same time point.

Abbreviations: Ox Alb, oxidized albumin; Nat Alb, native albumin; HUVEC, human umbilical vein endothelial cells.

\section{Discussion}

Endothelial dysfunction associated with cardiovascular diseases is a property related to aging. The mechanism by which endothelial cells undergo senescence is still largely unclear and yet to be discovered. Although this mechanism probably involves a multifactorial response, oxidative stress has been proposed as a mediator to explain the process of cellular senescence. Oxidative stress is characterized by excess free radical activity and plays an important role in the oxidation of proteins. Several studies have implicated the oxidation 
of low-density lipoprotein (LDL) in atherosclerosis. ${ }^{5,37,38}$ However, there is no evidence that relates the oxidized albumin, which is the most abundant protein in serum, with endothelial injury. Therefore, in this study, we investigated whether aging induced an increase in oxidized protein and whether oxidized albumin may be involved in aging-related endothelial damage.

The results of this study demonstrated that elderly subjects exhibit an increase of AOPPs and EMPs, reflecting endothelial damage and cellular senescence. To date, all the studies have analyzed the effect of oxidized LDL on endothelial damage. In plasma, the major protein presented is the albumin and LDL is present in lower concentrations, consequently, the albumin is more susceptible to suffer oxidation. In particular, there is no evidence showing that the oxidized albumin produced by oxidative stress generates cellular senescence. For this reason, our study is focused on oxidized albumin. Notwithstanding, plenty of data exist regarding the association between the presence of oxidized LDL presence and some senescence markers. ${ }^{39-41}$ It has also been described that AOPPs are carried by oxidized plasma proteins, especially albumin, and accumulate in subjects with renal disease and coronary artery disease. ${ }^{42}$ In this sense, these reported data indicate that oxidized proteins are increased in the plasma of elderly subjects.

In accordance, there are no findings demonstrating the presence of oxidized albumin in the plasma when there is endothelial dysfunction. In contrast, accumulating evidence suggest that the oxidation of LDL results in severe vascular damage. ${ }^{40,43}$ Here, we have shown an increase of the EMPs in the elderly subjects, suggesting that EMPs measurement is identified as a parameter in the endothelial damage. So, the in vitro model was used in this study to determine whether the oxidized albumin induces senescence and endothelial damage.

In this study, oxidized albumin-treated HUVECs cause the release of EMPs in the media and an increment of apoptosis levels. These findings support the idea that the endothelial cells are suffering from an endothelial activation, which is an apoptosis phenomenon not observed with native albumin treatment. Recent evidence also suggests that the endothelial cell is damaged as a consequence of cardiovascular disease. Furthermore, released EMPs are considered a marker of endothelial damage in patients. ${ }^{20,44}$ Several studies have demonstrated that adhesion molecules are secreted by activated endothelial cells and contributed to endothelial cell injury. ${ }^{26,27}$ Supporting this, our results demonstrate an increase of VCAM-1 and ICAM-1.

In addition, other studies have indicated the increase of modification proteins may be associated with oxidative stress development in aging. In this regard, there is a wealth of data evidencing the fact that protein modifications cause ROS production. ${ }^{37,45}$ As the results showed, oxidized albumin results in ROS production increment in endothelial cells as well as in the amount of ROS per cell (MFI increment). The enhancement of oxidative stress is considered a key mechanism in the cellular senescence development (replicative and premature induced by cardiovascular factors). ${ }^{40,46}$ In this study, the upregulation of ROS induced by oxidized albumin is correlated with an increase in the number of senescent cells. These data support the idea that the oxidized albumin may be considered a cardiovascular risk factor to induce oxidative stress. As a consequence, the cell may suffer senescence processes to prevent a possible damage due to oxidative stress. Additionally, we observed a slight ROS increment when the endothelial cells were treated with native albumin, which is not comparable with the elevated ROS levels observed with the oxidized albumin treatment; moreover, it is not associated with changes in other markers of cellular damage, such as adhesion molecules and apoptosis. In this sense, it seems that the ROS modification by native albumin is linked to normal cell proliferation and physiological signaling processes. Additional experiments are needed to consider the oxidized albumin quantification as a biological marker associated with endothelial damage and aging. Consequently, research is needed to explore the possibility of utilizing oxidized albumin as a potential therapeutic target.

Finally, we have shown that the migratory capacity of endothelial cells was reduced with oxidized albumin treatment. To date, experimental studies have found that oxidized LDL inhibits endothelial cell migration and may impair healing of arterial injuries ${ }^{47}$ In addition, this study reported that stimulation of ROS production by oxidized LDL inhibited endothelial cell migration. Likewise, oxidized albumin promotes intracellular ROS production and therefore is implicated in many cellular processes, including migration. As mentioned earlier, the endothelial senescence induced by oxidized albumin is a cellular mechanism to avoid ROS damage. Furthermore, this effect is associated with reduction in the endothelial migration activity.

\section{Conclusion}

Our data have shown that the plasma of elderly subjects contain oxidized proteins, especially oxidized albumin. The oxidation of this protein indicates an endothelial activation by adhesion molecule releases and the consequent endothelial damage through an oxidative stress and increase in apoptosis levels. Although further experiments are needed to better 
understand the role of oxidized albumin in endothelial injury, the identification of oxidized albumin as a possible marker for use in the clinic may be helpful in the diagnosis of the endothelial damage associated with aging. As a result, oxidized albumin may be utilized in prognosis and as a therapeutic target in preventing cardiovascular disease development.

\section{Acknowledgments}

We are grateful to María José Jimenez and Rosa Moyano for technical assistance. We also thank Miss Annie Bailey for English and scientific revisions of the manuscript. This work was supported by Plan Nacional Proyectos de Investigación en Salud of Instituto de Salud Carlos III (ISCIII) Fondos Feder European Grants (PI11/01536, PI12/01489, PI014/0041 and PI14/00806); Red de Investigación Renal (REDinREN; RD12/0021/002); Comunidad de Madrid (Fibroteam; S2010/BMD-2321); Junta de Andalucía Grants JA0797-2010, P010-CTS-6337, P11-CTS-7352.

\section{Author contributions}

Rafael Ramírez, Carlos Luna, Matilde Alique, and Estefania Navalmoral conceived and designed the experiments. Estefanía Navalmoral, Maria-Victoria Noci, Lourdes Bohorquez-Magro, and Julia Carracedo performed the experiments. Carlos Luna, Matilde Alique, and Julia Carracedo analyzed the data. Rafael Ramírez and Julia Carracedo contributed reagents/materials/analysis tools. Rafael Ramírez, Julia Carracedo, and Matilde Alique wrote the paper. All authors contributed toward data analysis, drafting and revising the paper and agree to be accountable for all aspects of the work.

\section{Disclosure}

Julia Carracedo is a fellow from Fundación de Investigaciones Biomédicas de Córdoba (Programa Nicolás Monardes). Carlos Luna is a fellow from Consejería de Innovación, Ciencia y Empresa, Junta de Andalucía (CTS-6337). Matilde Alique is a fellow of the program "Ayuda Postdoctoral Programa Propio" from Universidad de Alcala, Madrid, Spain. The authors report no other conflicts of interest in this work.

\section{References}

1. Levine RL, Stadtman ER. Oxidative modification of proteins during aging. Exp Gerontol. 2001;36(9):1495-1502.

2. Stadtman ER. Protein oxidation and aging. Free Radic Res. 2006; 40(12): 1250-1258.

3. Grune T, Reinheckel T, Davies KJ. Degradation of oxidized proteins in K562 human hematopoietic cells by proteasome. J Biol Chem. 1996; 271(26):15504-15509.

4. Rivett AJ. Regulation of intracellular protein turnover: covalent modification as a mechanism of marking proteins for degradation. Curr Top Cell Regul. 1986;28:291-337.
5. Gradinaru D, Borsa C, Ionescu C, Margina D. Advanced oxidative and glycoxidative protein damage markers in the elderly with type 2 diabetes. J Proteomics. 2013;92:313-322.

6. Stadtman ER, Levine RL. Protein oxidation. Ann N Y Acad Sci. 2000; 899:191-208.

7. Anderson NL, Anderson NG. The human plasma proteome: history, character, and diagnostic prospects. Mol Cell Proteomics. 2002;1(11): $845-867$.

8. Borges CR, Rehder DS, Jensen S, et al. Elevated plasma albumin and apolipoprotein A-I oxidation under suboptimal specimen storage conditions. Mol Cell Proteomics. 2014;13(7):1890-1899.

9. Berlett BS, Stadtman ER. Protein oxidation in aging, disease, and oxidative stress. J Biol Chem. 1997;272(33):20313-20316.

10. Dalle-Donne I, Giustarini D, Colombo R, Rossi R, Milzani A. Protein carbonylation in human diseases. Trends Mol Med. 2003;9(4): $169-176$.

11. Fan X, Zhang J, Theves M, et al. Mechanism of lysine oxidation in human lens crystallins during aging and in diabetes. J Biol Chem. 2009; 284(50):34618-34627.

12. Choi J, Forster MJ, McDonald SR, Weintraub ST, Carroll CA, Gracy RW. Proteomic identification of specific oxidized proteins in ApoE-knockout mice: relevance to Alzheimer's disease. Free Radic Biol Med. 2004;36(9):1155-1162.

13. Brennan ML, Hazen SL. Amino acid and protein oxidation in cardiovascular disease. Amino Acids. 2003;25(3-4):365-374.

14. De Marchi E, Baldassari F, Bononi A, Wieckowski MR, Pinton P. Oxidative stress in cardiovascular diseases and obesity: role of p66Shc and protein kinase C. Oxid Med Cell Longev. 2013;2013:564961.

15. Strobel NA, Fassett RG, Marsh SA, Coombes JS. Oxidative stress biomarkers as predictors of cardiovascular disease. Int J Cardiol. 2011; 147(2):191-201.

16. Brandes RP, Fleming I, Busse R. Endothelial aging. Cardiovasc Res. 2005;66(2):286-294.

17. Matz RL, Andriantsitohaina R. Age-related endothelial dysfunction: potential implications for pharmacotherapy. Drugs Aging. 2003;20(7): 527-550.

18. Davignon J, Ganz P. Role of endothelial dysfunction in atherosclerosis. Circulation. 2004;109(23 Suppl 1):III27-III32.

19. Banfi C, Brioschi M, Barcella S, et al. Oxidized proteins in plasma of patients with heart failure: role in endothelial damage. Eur J Heart Fail. 2008;10(3):244-251.

20. Buendía P, Montes de Oca A, Madueño JA, et al. Endothelial microparticles mediate inflammation-induced vascular calcification. FASEB J. 2015;29(1):173-181.

21. Bartoloni E, Alunno A, Bistoni O, et al. Characterization of circulating endothelial microparticles and endothelial progenitor cells in primary Sjögren's syndrome: new markers of chronic endothelial damage? Rheumatology (Oxford). 2015;54(3):536-544.

22. Lovren F, Verma S. Evolving role of microparticles in the pathophysiology of endothelial dysfunction. Clin Chem. 2013;59(8): $1166-1174$.

23. Fink K, Moebes M, Vetter C, et al. Selenium prevents microparticleinduced endothelial inflammation in patients after cardiopulmonary resuscitation. Crit Care. 2015;19:58.

24. Lee SK, Yang SH, Kwon I, Lee OH, Heo JH. Role of tumour necrosis factor receptor- 1 and nuclear factor- $\mathrm{\kappa B}$ in production of TNF- $\alpha$-induced pro-inflammatory microparticles in endothelial cells. Thromb Haemost. 2014;112(3):580-588.

25. Dignat-George F, Boulanger CM. The many faces of endothelial microparticles. Arterioscler Thromb Vasc Biol. 2011;31(1):27-33.

26. Zhu YP, Shen T, Lin YJ, et al. Astragalus polysaccharides suppress ICAM- 1 and VCAM-1 expression in TNF- $\alpha$-treated human vascular

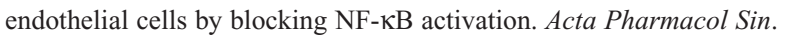
2013;34(8):1036-1042.

27. Al-Mutairi M, Al-Harthi S, Cadalbert L, Plevin R. Over-expression of mitogen-activated protein kinase phosphatase-2 enhances adhesion molecule expression and protects against apoptosis in human endothelial cells. Br J Pharmacol. 2010;161(4):782-798. 
28. van Deursen JM. The role of senescent cells in ageing. Nature. 2014; 509(7501):439-446.

29. Ibanez B, Giannarelli C, Cimmino G, et al. Recombinant HDL(Milano) exerts greater anti-inflammatory and plaque stabilizing properties than HDL(wild-type). Atherosclerosis. 2012;220(1):72-77.

30. Rubenstein DA, Maria Z, Yin W. Glycated albumin modulates endothelial cell thrombogenic and inflammatory responses. J Diabetes Sci Technol. 2011;5(3):703-713.

31. Herbert B, Galvani M, Hamdan M, et al. Reduction and alkylation of proteins in preparation of two-dimensional map analysis: why, when, and how? Electrophoresis. 2001;22(10):2046-2057.

32. Quagliaro L, Piconi L, Assaloni R, et al. Intermittent high glucose enhances ICAM-1, VCAM-1 and E-selectin expression in human umbilical vein endothelial cells in culture: the distinct role of protein kinase $\mathrm{C}$ and mitochondrial superoxide production. Atherosclerosis. 2005;183(2):259-267.

33. Yu H, Jiang W, Du H, et al. Involvement of the Akt/NF- $\mathrm{kB}$ pathways in the HTNV-mediated increase of IL-6, CCL5, ICAM-1, and VCAM-1 in HUVECs. PLoS One. 2014;9(4):e93810.

34. Winn RK, Harlan JM. The role of endothelial cell apoptosis in inflammatory and immune diseases. J Thromb Haemost. 2005;3(8):1815-1824.

35. Carracedo J, Buendía P, Merino A, et al. Cellular senescence determines endothelial cell damage induced by uremia. Exp Gerontol. 2013; 48(8):766-773.

36. Wagner M, Hampel B, Bernhard D, Hala M, Zwerschke W, Jansen-Dürr P. Replicative senescence of human endothelial cells in vitro involves G1 arrest, polyploidization and senescence-associated apoptosis. Exp Gerontol. 2001;36(8):1327-1347.

37. Chuang CY, Degendorfer G, Hammer A, Whitelock JM, Malle E, Davies MJ. Oxidation modifies the structure and function of the extracellular matrix generated by human coronary artery endothelial cells. Biochem J. 2014;459(2):313-322.

38. Schaer CA, Deuel JW, Bittermann AG, et al. Mechanisms of haptoglobin protection against hemoglobin peroxidation triggered endothelial damage. Cell Death Differ. 2013;20(11):1569-1579.
39. Uchida K, Kanematsu M, Sakai K, et al. Protein-bound acrolein: potential markers for oxidative stress. Proc Natl Acad Sci USA. 1998;95(9): 4882-4887.

40. Shi Y, Lüscher TF, Camici GG. Dual role of endothelial nitric oxide synthase in oxidized LDL-induced, p66Shc-mediated oxidative stress in cultured human endothelial cells. PLoS One. 2014;9(9):e107787.

41. Carracedo J, Merino A, Briceño C, et al. Carbamylated low-density lipoprotein induces oxidative stress and accelerated senescence in human endothelial progenitor cells. FASEB J. 2011;25(4):1314-1322.

42. Marsche G, Frank S, Hrzenjak A, et al. Plasma-advanced oxidation protein products are potent high-density lipoprotein receptor antagonists in vivo. Circ Res. 2009;104(6):750-757.

43. Huang CS, Lin AH, Liu CT, et al. Isothiocyanates protect against oxidized LDL-induced endothelial dysfunction by upregulating Nrf2dependent antioxidation and suppressing NFKB activation. Mol Nutr Food Res. 2013;57(11):1918-1930.

44. Schiro A, Wilkinson FL, Weston R, Smyth JV, Serracino-Inglott F, Alexander MY. Endothelial microparticles as conveyors of information in atherosclerotic disease. Atherosclerosis. 2014;234(2):295-302.

45. Wu J, He Z, Gao X, et al. Oxidized high-density lipoprotein impairs endothelial progenitor cells' function by activation of CD36-MAPKTSP-1 pathways. Antioxid Redox Signal. 2015;22(4):308-324.

46. Buendía P, Carracedo J, Soriano S, et al. Klotho prevents NFKB translocation and protects endothelial cell from senescence induced by uremia. J Gerontol A Biol Sci Med Sci. 2015;70(10):1198-1209.

47. van Aalst JA, Zhang DM, Miyazaki K, Colles SM, Fox PL, Graham LM. Role of reactive oxygen species in inhibition of endothelial cell migration by oxidized low-density lipoprotein. J Vasc Surg. 2004;40(6): $1208-1215$.
Clinical Interventions in Aging

\section{Publish your work in this journal}

Clinical Interventions in Aging is an international, peer-reviewed journal focusing on evidence-based reports on the value or lack thereof of treatments intended to prevent or delay the onset of maladaptive correlates of aging in human beings. This journal is indexed on PubMed Central, MedLine,

\section{Dovepress}

CAS, Scopus and the Elsevier Bibliographic databases. The manuscript management system is completely online and includes a very quick and fair peer-review system, which is all easy to use. Visit http://www.dovepress. com/testimonials.php to read real quotes from published authors. 